Original Research Paper

\title{
Multistep Collocation Block Method for Direct Solution of Second Order Ordinary Differential Equations
}

\author{
John Olusola Kuboye and Zurni Omar \\ Department of Mathematics, School of Quantitative Sciences, \\ College of Art and Sciences, Universiti Utara Sintok, Kedah, Malaysia
}

Article history

Received: 10-08-2015

Revised: 28-09-2015

Accepted: 29-09-2015

Corresponding Author: John Olusola Kuboye

Department of Mathematics,

School of Quantitative

Sciences, College of Art and

Sciences, Universiti Utara

Sintok, Kedah, Malaysia

Email: kubbysholly2007@gmail.com

\begin{abstract}
In this study, block method of order six for solving second order ordinary differential equations is developed. The method of interpolation of approximated power series and collocation of its second derivative is adopted in the derivation of the method. The developed method is applied in block form to produce approximate solution at five points simultaneously. The stability properties of the method such as order, error constants, consistency and convergence are investigated. The developed method was then applied to solve some initial value problems of second order ordinary differential equations and the numerical results indicated that the new method produced better accuracy than the existing methods when solving the same problems.
\end{abstract}

Keywords: Power Series, Multistep Collocation, Second Order Initial Value Problems

\section{Introduction}

In the field of science and engineering, mathematical models are usually developed to help in the understanding of physical phenomena. These models are always resulted to differential equations. Ross (1989) stated some of the problems that involved differential equations as follows:

$>$ The problem arising from determining the projectile motion, satellite, rocket or planet

$>$ The problem of how to determine the charge or current in an electric circuit

$>$ The study of chemical reactions

$>$ The study of decomposition rate of radioactive substance or population growth rate

$>$ The problem of the conduction in a rod or in a slab

$>$ The problem of determining the vibrations of a wire or membrane

These problems highlighted above obey certain scientific laws that involve rates of change of one or more quantities. Mathematically, these rates of change can be expressed by derivatives (Omar, 1999). When the problems are converted to mathematical equations they will form differential equations. The third order ordinary differential equations of the form:

$$
y^{\prime \prime}=f\left(x, y, y^{\prime}\right), y(a)=y_{0}, y^{\prime}(a)=y^{\prime}(a)=y_{0}^{\prime}, x \in[a, b]
$$

is considered in this study. Shortly after the introduction of calculus, it was discovered that not all Ordinary Differential Equations (ODEs) can be solved analytically. In order to overcome this great challenge, numerical methods were developed with the aim of providing approximate solution to the ODEs and one of these methods is predictor-corrector method. The development of linear multistep method has been examined by scholars such as Awoyemi (2001), Omolehin et al. (2003) and Kayode and Adeyeye (2013) for solving (1) directly. These researchers developed numerical methods which were implemented in predictor-corrector mode where Taylor series algorithm is used to supply the starting values. The major setback of this method is the cost of developing predictors. Moreover, the predictors developed are of lower order to the correctors. Hence, this brings lower accuracy on the generated results (Adesanya et al., 2013).

In order to overcome the rigor in developing separate predictors and other shortcoming in predictorcorrector method, the development of block method was proposed. This computes the discrete method at more than one grid point simultaneously. According to Olabode (2007) block method was first proposed by Milne in 1953 who advocated the use of block as a means of getting a starting value for predictor-corrector algorithm and later adopted as a full method (Anake et al., 2001; Adesanya et al., 2013). 
Researchers like Ehigie et al. (2011), Jator (2007) and Awari et al. (2014) developed block methods without predictors for solving (1) directly. Furthermore, block methods with step-length $k=5$ were developed by Mohammed and Adeniyi (2014), Mohammed (2011) and Badmus and Yahaya (2009). It is observed that the methods are of lower accuracy.

For the purpose of comparing with the existing methods of the same step-length $k=5$ and also bring improvement on numerical methods, this paper presents new block method with step-length $k=5$ for solving second order initial value problems directly. The points of interpolation were made at $x=x_{n+i}, i=2,3$ and collocation points $x=x_{n+i}, i=0(1) k$.

\section{Methodology}

We consider power series of the form:

$$
y(x)=\sum_{j=0}^{k+2} a_{j} x^{j}
$$

as an approximate solution to (1). Where $k=5$. The first and second derivative of (2) gives:

$$
\begin{aligned}
& y^{\prime}(x)=\sum_{j=1}^{k+2} j a_{j} x^{j-1} \\
& y^{\prime \prime}(x)=\sum_{j=2}^{k+2} j(j-1) a_{j} x^{j-2}=f\left(x, y, y^{\prime}\right)
\end{aligned}
$$

Equation (2) is interpolated at the points $x=x_{n+i}, i=$ 2,3 and (4) is collocated at $x=x_{n+i}, i=0(1) 5$, this produces nonlinear equations of the form:

$$
\left(\begin{array}{cccccccc}
1 & x_{n+2} & x_{n+2}^{2} & x_{n+2}^{3} & x_{n+2}^{4} & x_{n+2}^{5} & x_{n+2}^{6} & x_{n+2}^{7} \\
1 & x_{n+3} & x_{n+3}^{2} & x_{n+3}^{3} & x_{n+3}^{4} & x_{n+3}^{5} & x_{n+3}^{6} & x_{n+3}^{7} \\
0 & 0 & 2 & 6 x_{n} & 12 x_{n}^{2} & 20 x_{n}^{3} & 30 x_{n}^{4} & 42 x_{n}^{5} \\
0 & 0 & 2 & 6 x_{n+1} & 12 x_{n+1}^{2} & 20 x_{n+1}^{3} & 30 x_{n+1}^{4} & 42 x_{n+1}^{5} \\
0 & 0 & 2 & 6 x_{n+2} & 12 x_{n+2}^{2} & 20 x_{n+2}^{3} & 30 x_{n+2}^{4} & 42 x_{n+2}^{5} \\
0 & 0 & 2 & 6 x_{n+3} & 12 x_{n+3}^{2} & 20 x_{n+3}^{3} & 30 x_{n+3}^{4} & 42 x_{n+3}^{5} \\
0 & 0 & 2 & 6 x_{n+4} & 12 x_{n+4}^{2} & 20 x_{n+4}^{3} 30 x_{n+4}^{4} 42 x_{n+4}^{5} \\
0 & 0 & 2 & 6 x_{n+5} & 12 x_{n+5}^{2} & 20 x_{n+5}^{3} & 30 x_{n+5}^{4} & 42 x_{n+5}^{5}
\end{array}\right)\left(\begin{array}{l}
a_{0} \\
a_{1} \\
a_{2} \\
a_{3} \\
a_{4} \\
a_{5} \\
a_{6} \\
a_{7}
\end{array}\right)=\left(\begin{array}{l}
y_{n+2} \\
y_{n+3} \\
f_{n} \\
f_{n+1} \\
f_{n+2} \\
f_{n+3} \\
f_{n+4} \\
f_{n+5}
\end{array}\right)(5)
$$

In finding the values of $a$ 's in (5), Gaussian elimination is employed. Substituting the values of $a$ 's (refer to Appendix A) into Equation (2) and simplifying, this gives a continuous linear multistep method of the form:

$$
y(x)=\sum_{j=2}^{k-2} a_{j}(x) y_{n+j}+h^{2} \sum_{j=0}^{k} \beta_{0}(x) f_{n+j}
$$

Where:

$$
x=z h+x_{n}+4 h
$$

Substituting (7) in (6) and simplifying, we have the following coefficients of $y_{n+j}$ and $f_{n+j}$ as:

$$
\left(\begin{array}{ll}
a_{2} & (z) \\
a_{3} & (z)
\end{array}\right)=\left(\begin{array}{cc}
-1 & -1 \\
2 & 1
\end{array}\right)\left(\begin{array}{l}
z^{0} \\
z
\end{array}\right)
$$

$$
\left(\begin{array}{l}
\beta_{1}(z) \\
\beta_{2}(z) \\
\beta_{3}(z) \\
\beta_{4}(z) \\
\beta_{5}(z)
\end{array}\right)=D\left(\begin{array}{l}
z^{0} \\
z^{1} \\
z^{2} \\
z^{3} \\
z^{4} \\
z^{5} \\
z^{6} \\
z^{7}
\end{array}\right)
$$

Where:

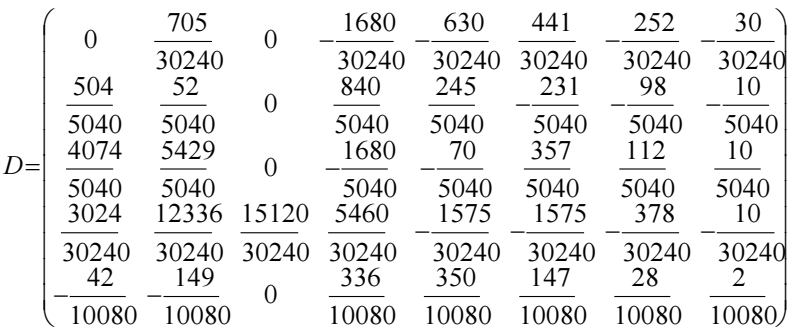

Evaluating (8) and (9) at the non-interpolating points that is, at $z=-4,-3,0$ and 1 .

This produces the following schemes:

$$
\left(\begin{array}{c}
y_{n} \\
y_{n+1} \\
y_{n+4} \\
y_{n+5}
\end{array}\right)=\left(\begin{array}{cc}
-2 & 3 \\
2 & -1 \\
-1 & 2 \\
-2 & 3
\end{array}\right)\left(\begin{array}{l}
y_{n+2} \\
y_{n+3}
\end{array}\right)+h^{2} E\left(\begin{array}{l}
f_{n} \\
f_{n+1} \\
f_{n+2} \\
f_{n+3} \\
f_{n+4} \\
f_{n+5}
\end{array}\right)
$$

Where:

$$
E=\left(\begin{array}{cccccc}
\frac{1}{240} & \frac{-8}{240} & \frac{62}{240} & \frac{392}{240} & \frac{257}{240} & \frac{16}{240} \\
\frac{-1}{240} & \frac{24}{240} & \frac{194}{240} & \frac{24}{240} & \frac{-1}{240} & 0 \\
0 & \frac{-1}{240} & \frac{24}{240} & \frac{194}{240} & \frac{24}{240} & \frac{-1}{240} \\
\frac{1}{240} & \frac{-8}{240} & \frac{62}{240} & \frac{392}{240} & \frac{257}{240} & \frac{16}{240}
\end{array}\right)
$$

The values of $y_{n+2}$ and $y_{n+3}$ are represented in Taylor series of the form: 


$$
\begin{aligned}
& y_{n+2}=\sum_{m=0}^{k+2} \frac{(2 h)^{m}}{m !} y_{n}^{(m)} \\
& y_{n+3}=\sum_{m=0}^{k+2} \frac{(3 h)^{m}}{m !} y_{n}^{(m)}
\end{aligned}
$$

The derivative of (8) and (9) gives:

$$
\left(\begin{array}{ll}
a_{2}^{\prime} & (z) \\
a_{3}^{\prime} & (z)
\end{array}\right)\left(\begin{array}{c}
-1 \\
1
\end{array}\right)
$$

$$
\left(\begin{array}{l}
\beta_{0}^{\prime}(z) \\
\beta_{1}^{\prime}(z) \\
\beta_{2}^{\prime}(z) \\
\beta_{3}^{\prime}(z) \\
\beta_{4}^{\prime}(z) \\
\beta_{5}^{\prime}(z)
\end{array}\right)=F\left(\begin{array}{c}
z^{0} \\
z^{1} \\
z^{2} \\
z^{3} \\
z^{4} \\
z^{5} \\
z^{6} \\
z^{7}
\end{array}\right)
$$

For:

$$
F=\left(\begin{array}{ccccccc}
-\frac{40}{10080} & 0 & \frac{252}{10080} & \frac{140}{10080} & -\frac{105}{10080} & -\frac{84}{10080} & -\frac{14}{10080} \\
\frac{235}{10080} & 0 & -\frac{1680}{10080} & -\frac{840}{10080} & \frac{735}{10080} & \frac{504}{10080} & \frac{70}{10080} \\
\frac{52}{5040} & 0 & \frac{2520}{5040} & \frac{980}{5040} & -\frac{1155}{5040} & -\frac{588}{5040} & -\frac{70}{5040} \\
\frac{5429}{5040} & 0 & -1 & -\frac{280}{5040} & \frac{1785}{5040} & \frac{672}{5040} & \frac{70}{5040} \\
\frac{4112}{10080} & 1 & \frac{5460}{10080} & -\frac{2100}{10080} & -\frac{2625}{10080} & -\frac{756}{10080} & -\frac{70}{10080} \\
-\frac{149}{10080} & 0 & 1 & \frac{1400}{10080} & \frac{735}{10080} & \frac{168}{10080} & \frac{14}{10080}
\end{array}\right)
$$

Equations (11) and (12) are evaluated at all the grid points. That is, at $z=-4,-3,-2,-1,0$ and 1 . This produces the following schemes:

$$
\left(\begin{array}{l}
y_{n+1}^{\prime} \\
y_{n+2}^{\prime} \\
y_{n+3}^{\prime} \\
y_{n+4}^{\prime} \\
y_{n+5}^{\prime}
\end{array}\right)=\frac{1}{h}\left(\begin{array}{cc}
1 & -1 \\
1 & -1 \\
1 & -1 \\
1 & -1 \\
1 & -1
\end{array}\right)\left(\begin{array}{c}
y_{n+2} \\
y_{n+3}
\end{array}\right)+\frac{h}{10080} G\left(\begin{array}{c}
f_{n} \\
f_{n+1} \\
f_{n+2} \\
f_{n+3} \\
f_{n+4} \\
f_{n+5}
\end{array}\right)
$$

Where:

$$
G=\left(\begin{array}{cccccc}
149 & -4112 & -10858 & -104 & -235 & 40 \\
-40 & 347 & -3704 & -1910 & 304 & -37 \\
37 & -304 & 1910 & 3704 & -347 & 40 \\
-40 & 235 & 104 & 10858 & 4112 & -149 \\
149 & -976 & 3478 & 5272 & 14101 & 3176
\end{array}\right)
$$

\section{Analysis of the Method}

\section{Analysis of the Method}

In finding the order of the method, the method proposed by Lambert (1973) is adopted. That is taking the Taylor series of (10) at point $x_{n}$ for $y_{n+1}, y_{n+4}$ and $y_{n+5}$ gives L (refer to Appendix B).

Therefore, comparing the coefficients of $h^{m}$ and $y_{n}^{m}$. This gives our method to have a uniform order $(6,6,6)$ with error constants $\left(\frac{31}{60480}, \frac{31}{60480},-\frac{53}{20160}\right)$. Since the order of the method is greater than one, this implies the method is consistent.

\section{Numerical Experiments}

In order to examine the accuracy of the new method, the following second order initial value problems are put into consideration. The results generated are shown in Tables 1-5:

Problem 1: $y^{\prime \prime}=y^{\prime}, y(0)=0, y^{\prime}(0)=-1, h=0.1$

Exact Solution: $y(x)=1-e^{x}$

Table 1. The exact solution and computed solution of the new method for problem 1

\begin{tabular}{lll}
\hline & $\begin{array}{l}\text { Exact solution } \\
{[\mathrm{y}(\mathrm{x})]}\end{array}$ & $\begin{array}{l}\text { Computed solution } \\
{\left[\mathrm{y}\left(\mathrm{x}_{\mathrm{n}}\right)\right]}\end{array}$ \\
\hline 0.1 & -0.1051709 & -0.1051710 \\
0.2 & -0.2214028 & -0.2214028 \\
0.3 & -0.3498588 & -0.3498588 \\
0.4 & -0.4918247 & -0.4918246 \\
0.5 & -0.6487213 & -0.6487211 \\
0.6 & -0.8221188 & -0.8221185 \\
0.7 & -1.0137527 & -1.0137521 \\
0.8 & -1.2255409 & -1.2255400 \\
0.9 & -1.4596031 & -1.4596018 \\
0.1 & -1.7182818 & -1.7182801 \\
\hline
\end{tabular}

Table 2. Comparison of the new method with Mohammed and Adeniyi (2014) block hybrid backward difference formula and Mohammed (2011) block method for problem 1

\begin{tabular}{llll}
\hline & $\begin{array}{l}\text { Error in new } \\
\text { Method, } k=5 \\
{\left[\mathrm{y}(\mathrm{x})-\mathrm{y}\left(\mathrm{x}_{\mathrm{n}}\right)\right]}\end{array}$ & $\begin{array}{l}\text { Error in Mohammed } \\
\text { and Adeniyi } \\
(2014), k=5\end{array}$ & $\begin{array}{l}\text { Error } \\
\text { in Mohammed } \\
(2011), k=5\end{array}$ \\
\hline 0.1 & $3.57 \mathrm{E}-08$ & $2.00 \mathrm{E}-07$ & $2.20 \mathrm{E}-05$ \\
0.2 & $2.60 \mathrm{E}-09$ & $5.39 \mathrm{E}-07$ & $6.07 \mathrm{E}-06$ \\
0.3 & $4.51 \mathrm{E}-08$ & $8.84 \mathrm{E}-07$ & $1.01 \mathrm{E}-05$ \\
0.4 & $9.23 \mathrm{E}-08$ & $1.23 \mathrm{E}-06$ & $1.40 \mathrm{E}-05$ \\
0.5 & $1.48 \mathrm{E}-07$ & $1.58 \mathrm{E}-06$ & $1.80 \mathrm{E}-05$ \\
0.6 & $3.06 \mathrm{E}-07$ & $1.92 \mathrm{E}-06$ & $2.16 \mathrm{E}-05$ \\
0.7 & $6.08 \mathrm{E}-07$ & $2.51 \mathrm{E}-06$ & $2.80 \mathrm{E}-05$ \\
0.8 & $9.43 \mathrm{E}-07$ & $3.11 \mathrm{E}-06$ & $3.46 \mathrm{E}-05$ \\
0.9 & $1.31 \mathrm{E}-06$ & $3.71 \mathrm{E}-06$ & $4.11 \mathrm{E}-05$ \\
0.1 & $1.73 \mathrm{E}-06$ & $4.30 \mathrm{E}-06$ & $4.77 \mathrm{E}-05$ \\
\hline
\end{tabular}


Problem 2: $y^{\prime \prime}+\left(\frac{6}{x}\right) y^{\prime}+\left(\frac{4}{x^{2}}\right) y=0, \quad y(1)=1$,

$$
y^{\prime}(1)=1, h=\frac{0.1}{32}
$$

Exact Solution: $y(x)=\frac{5 x^{3}-2}{3 x^{4}}$

Table 3. The exact solution and computed solution of the new method for problem 2

\begin{tabular}{lll}
\hline x-values & $\begin{array}{l}\text { Exact solution } \\
{[\mathrm{y}(\mathrm{x})]}\end{array}$ & $\begin{array}{l}\text { Computed solution } \\
{\left[\mathrm{y}\left(\mathrm{x}_{\mathrm{n}}\right)\right]}\end{array}$ \\
\hline 0.003125 & 1.0030765 & 1.0030765 \\
0.006250 & 1.0060575 & 1.0060575 \\
0.009375 & 1.0089450 & 1.0089450 \\
0.012500 & 1.0117410 & 1.0117410 \\
0.015625 & 1.0144475 & 1.0144475 \\
0.018750 & 1.0170665 & 1.0170665 \\
0.025000 & 1.0220492 & 1.0220492 \\
0.028125 & 1.0244165 & 1.0244165 \\
0.031250 & 1.0267036 & 1.0267036 \\
\hline
\end{tabular}

Table 4. Comparison of the new method with Badmus and Yahaya (2009) block method for solving Problem 2

\begin{tabular}{lll}
\hline & $\begin{array}{l}\text { Error in new } \\
\text { Method, } k=5 \\
{\left[\mathrm{y}(\mathrm{x})-\mathrm{y}\left(\mathrm{x}_{\mathrm{n}}\right)\right]}\end{array}$ & $\begin{array}{l}\text { Error in Badmus } \\
\text { and Yahaya, } \\
(2009), k=5\end{array}$ \\
\hline 0.003125 & $3.06 \mathrm{E}-11$ & $3.84 \mathrm{E}-05$ \\
0.006250 & $3.21 \mathrm{E}-12$ & $7.50 \mathrm{E}-05$ \\
0.009375 & $3.64 \mathrm{E}-11$ & $1.06 \mathrm{E}-04$ \\
0.012500 & $6.90 \mathrm{E}-11$ & $1.35 \mathrm{E}-04$ \\
0.015625 & $1.01 \mathrm{E}-10$ & $1.56 \mathrm{E}-04$ \\
0.018750 & $9.38 \mathrm{E}-11$ & $1.86 \mathrm{E}-04$ \\
0.025000 & $1.88 \mathrm{E}-10$ & $1.96 \mathrm{E}-04$ \\
0.028125 & $2.34 \mathrm{E}-10$ & $2.21 \mathrm{E}-04$ \\
0.031250 & $2.79 \mathrm{E}-10$ & $2.06 \mathrm{E}-04$ \\
\hline
\end{tabular}

Problem 3: $y^{\prime \prime}=-y+2 \cos x \quad y(0)=1$,

$$
y^{\prime}(0)=0,0 \leq x \leq 1
$$

Exact Solution: $y(x)=\cos x+x \sin x$

Table 5. Comparison of the new method with Omar (2004) block method in which maximum errors were considered for Problem 3

\begin{tabular}{lllll}
\hline $\mathrm{h}$ & $\begin{array}{l}\text { Omar } \\
(2004)\end{array}$ & $\begin{array}{l}\text { Number } \\
\text { of steps }\end{array}$ & $\begin{array}{l}\text { Error in new } \\
\text { Method, } k=5\end{array}$ & $\begin{array}{c}\text { Error in Omar } \\
(2004), k=5\end{array}$ \\
\hline $10^{-2}$ & S2PEB & 53 & $2.59 \mathrm{E}-09$ & $1.43 \mathrm{E}-03$ \\
& P2PEB & 53 & $2.59 \mathrm{E}-09$ & $1.43 \mathrm{E}-03$ \\
$10^{-3}$ & S3PEB & 36 & $7.11 \mathrm{E}-09$ & $1.43 \mathrm{E}-03$ \\
& P3PEB & 36 & $7.11 \mathrm{E}-09$ & $1.43 \mathrm{E}-03$ \\
& S2PEB & 503 & $3.43 \mathrm{E}-12$ & $1.43 \mathrm{E}-04$ \\
& P2PEB & 503 & $3.43 \mathrm{E}-12$ & $1.43 \mathrm{E}-04$ \\
& S3PEB & 336 & $9.69 \mathrm{E}-13$ & $1.43 \mathrm{E}-04$ \\
$10^{-4}$ & P3PEB & 336 & $9.69 \mathrm{E}-13$ & $1.43 \mathrm{E}-04$ \\
& S2PEB & 5003 & $1.85 \mathrm{E}-10$ & $1.43 \mathrm{E}-05$ \\
& P2PEB & 5003 & $1.85 \mathrm{E}-10$ & $1.43 \mathrm{E}-05$ \\
& S3PEB & 3336 & $3.87 \mathrm{E}-10$ & $1.43 \mathrm{E}-05$ \\
$10^{-5}$ & P3PEB & 3336 & $3.87 \mathrm{E}-10$ & $1.43 \mathrm{E}-05$ \\
& S2PEB & 50003 & $1.82 \mathrm{E}-08$ & $1.43 \mathrm{E}-06$ \\
& P2PEB & 50003 & $1.82 \mathrm{E}-08$ & $1.43 \mathrm{E}-06$ \\
& S3PEB & 33336 & $1.00 \mathrm{E}-08$ & $1.43 \mathrm{E}-06$ \\
& P3PEB & 33336 & $1.00 \mathrm{E}-08$ & $1.43 \mathrm{E}-06$ \\
\hline
\end{tabular}

\section{Conclusion}

The derivation of block method of order six with step-length $k=5$ for solving second order ordinary differential equations has been considered in this study. The application of the new method to second order initial value problems confirmed the superiority of the method when compared with the existing methods of the same step-length $k=5$ (Refer to Table 2, 4 and 5).

\section{Acknowledgement}

The authors appreciate the Universiti Utara Malaysia for the financial support rendered to this research work.

\section{Author's Contributions}

Both authors have made good contributions to the development and publication of this manuscript.

\section{Conflict of Interests}

The authors declare that there is no conflict of interests regarding the publication of this paper.

\section{References}

Adesanya, A.O., M.R. Odekunle and M.O. Udoh, 2013. Four steps continuous method for the solution of $y^{\prime \prime}=f\left(x, y, y^{\prime}\right)$. Am. J. Computational Math., 3: 169-174. DOI: 10.4236/ajcm.2013.32025

Anake, T.A., D.O. Awoyemi and A.A. Adesanya, 2001. A one step method for the solution of general second order ordinary differential Equations. Int. J. Sci. Technol., 2: 159-163.

Awari, Y.S., E.E. Chima, N.M. Kamoh and F.L. Oladele, 2014. A family of implicit uniformly accurate order block integrators for the solution of second order ordinary differential equations. Int. J. Math. Statistic Invention, 2: 33-46.

Awoyemi, D.O., 2001. A new sixth-order algorithm for general second order ordinary differential equations. Int. J. Comput. Math., 77: 117-124. DOI: 10.1080/00207160108805054

Badmus, A.M. and Y.A. Yahaya, 2009. An accurate uniform order 6 block method for direct solution of general second order ordinary differential equations. Pacific J. Sci. Technol., 10: 248-254.

Ehigie, J.O., S.A. Okunuga and A.B. Sofoluwe, 2011. 3-point block methods for direct integration of general second-order ordinary differential equations. Advances Numerical Analysis, 10: 1-14. DOI: $10.1155 / 2011 / 513148$

Jator, S.N., 2007. A sixth order linear multistep method for the direct solution of $y^{\prime \prime}=f\left(x, y, y^{\prime}\right)$. Int. J. Pure Applied Math., 40: 457-472. 
Kayode, S.J. and O. Adeyeye, 2013. A 2-step two-point hybrid methods for direct solution of second order initial value problems. Afric. J. Math. Comp. Sci., 6: 191-196.

Lambert, J.D., 1973. Computational Methods in Ordinary Differential Equations. Introductory Mathematics for Scientists and Engineers. Wiley.

Mohammed, U., 2011. A class of implicit five-step block method for general second order ordinary differential equations. J. Nigeria Math. Society, 30: 25-39.

Mohammed, U. and R.B. Adeniyi, 2014. Derivation of Five Step Block Hybrid Backward Differential Formulas (HBDF) through the continuous multi-step collocation for solving second order differential equation. Pacific J. Sci. Technol., 15: 89-95.

Olabode, B.T., 2007. Some linear multistep methods for special and general third order initial value problems of ordinary differential equation. $\mathrm{PhD}$ Thesis, Federal University of Technology, Akure. (Unpublished,).

Omar, Z., 1999. Parallel block methods for solving higher order ordinary differential equations directly. PhD Thesis, Faculty of Science and Environmental studies Universiti Putra Malaysia.

Omolehin, J.O., M.A. Ibiejugba, M.O. Alabi and D.J. Evans, 2003. A new class of adams-bashforth schemes for ODEs. Int. J. Comput. Math., 80: 629-638. DOI: 10.1080/0020716021000023051

Ross, S.L., 1989. Introduction to Ordinary Differential Equations. 4th Edn., John Wiley and Sons, Inc, Singapore.

\section{Appendix A}

The values of $a$ 's are below:

$$
\begin{aligned}
& a_{0}=\quad 3 y_{n+2}-2 y_{n+3}+\frac{1}{15} h^{2} f_{n}+\frac{257}{240} h^{2} f_{n+1}+\frac{49}{30} h^{2} f_{n+2}+\frac{31}{120} h^{2} f_{n+3}-\frac{1}{30} h^{2} f_{n+4}+ \\
& \frac{1}{240} h^{2} f_{n+5}+\frac{1}{2} x_{n}^{2} f_{n}+\frac{x_{n}^{6}}{720 h^{4}}\left(3 f_{n}-14 f_{n+1}+26 f_{n+2}-24 f_{n+3}+11 f_{n+4}-2 f_{n+5}\right)+ \\
& \frac{x_{n}^{5}}{480 h^{3}}\left(17 f_{n}-71 f_{n+1}+118 f_{n+2}-98 f_{n+3}+41 f_{n+4}-7 f_{n+5}\right)+\frac{x_{n}^{4}}{288 h^{2}}\left(45 f_{n}-\right. \\
& \left.154 f_{n+1}+214 f_{n+2}-156 f_{n+3}+61 f_{n+4}-10 f_{n+5}\right)+\frac{x_{n}}{h}\left(y_{n+2}-y_{n+3}\right)+\frac{x_{n}^{3}}{360 h}\left(137 f_{n}\right. \\
& \left.-300 f_{n+1}+300 f_{n+2}-200 f_{n+3}+75 f_{n+4}-12 f_{n+5}\right)+\frac{x_{n}^{7}}{5040 h^{5}}\left(f_{n}-5 f_{n+1}+10 f_{n+2}\right. \\
& \left.-10 f_{n+3}+5 f_{n+4}-f_{n+5}\right)+\frac{397 h}{1260} x_{n} f_{n}+\frac{14101 h}{10080} x_{n} f_{n+1}+\frac{659 h}{1260} x_{n} f_{n+2}+\frac{1739 h}{5040} x_{n} f_{n+3} \\
& +\frac{397 h}{1260} x_{n} f_{n}+\frac{14101 h}{10080} x_{n} f_{n+1}+\frac{659 h}{1260} x_{n} f_{n+2}+\frac{1739 h}{5040} x_{n} f_{n+3}-\frac{61 h}{630} x_{n} f_{n+4}+\frac{149 h}{10080} x_{n} f_{n+5} \\
& a_{1}=\quad \frac{61}{630} h f_{n+4}-\frac{14101}{10080} h f_{n+1}-\frac{659}{1260} h f_{n+2}-\frac{1739}{5040} h f_{n+3}-\frac{397}{1260} h f_{n}-\frac{149}{10080} h f_{n+5}-x_{n} f_{n}- \\
& \frac{1}{h}\left(y_{n+2}-y_{n+3}\right)-\frac{x_{n}^{2}}{120 h}\left(137 f_{n}-300 f_{n+1}+300 f_{n+2}-200 f_{n+3}+75 f_{n+4}-12 f_{n+5}\right)-\frac{x_{n}^{5}}{120 h^{4}}\left(3 f_{n}\right. \\
& \left.-14 f_{n+1}+26 f_{n+2}-24 f_{n+3}+11 f_{n+4}-2 f_{n+5}\right)-\frac{x_{n}^{4}}{96 h^{3}}\left(17 f_{n}-71 f_{n+1}+118 f_{n+2}-98 f_{n+3}+41 f_{n+4}\right. \\
& \left.-7 f_{n+5}\right)-\frac{x_{n}^{3}}{72 h^{2}}\left(45 f_{n}-154 f_{n+1}+214 f_{n+2}-156 f_{n+3}+61 f_{n+4}-10 f_{n+5}\right)-\frac{x_{n}^{6}}{720 h^{5}}\left(f_{n}-5 f_{n+1}+\right. \\
& \left.10 f_{n+2}-10 f_{n+3}+5 f_{n+4}-f_{n+5}\right) \text {. } \\
& a_{2}=\quad \frac{1}{2} f_{n}+\frac{x_{n}^{4}}{48 h^{4}}\left(3 f_{n}-14 f_{n+1}+26 f_{n+2}-24 f_{n+3}+11 f_{n+4}-2 f_{n+5}\right)+\frac{x_{n}^{3}}{48 h^{3}}\left(17 f_{n}-71 f_{n+1}+\right. \\
& \left.118 f_{n+2}-98 f_{n+3}+41 f_{n+4}-7 f_{n+5}\right)+\frac{x_{n}^{2}}{48 h^{2}}\left(45 f_{n}-154 f_{n+1}+214 f_{n+2}-156 f_{n+3}+61 f_{n+4}-\right. \\
& \left.0 f_{n+5}\right)+\frac{x_{n}}{120 h}\left(137 f_{n}-300 f_{n+1}+300 f_{n+2}-200 f_{n+3}+75 f_{n+4}-12 f_{n+5}\right)+\frac{x_{n}^{5}}{240 h^{5}}\left(f_{n}-5 f_{n+1}\right. \\
& \left.+10 f_{n+2}-10 f_{n+3}+5 f_{n+4}-f_{n+5}\right) \text {. }
\end{aligned}
$$




$$
\begin{aligned}
& a_{3}=\quad \frac{-137}{360 h} f_{n}+\frac{5}{6 h} f_{n+1}-\frac{5}{6 h} f_{n+2}+\frac{5}{9 h} f_{n+3}-\frac{5}{24 h} f_{n+4}+\frac{1}{30 h} f_{n+5}-\frac{x_{n}^{3}}{36 h^{4}}\left(3 f_{n}-14 f_{n+1}+\right. \\
& \left.26 f_{n+2}-24 f_{n+3}+11 f_{n+4}-2 f_{n+5}\right)-\frac{x_{n}^{2}}{48 h^{3}}\left(17 f_{n}-71 f_{n+1}+118 f_{n+2}-98 f_{n+3}+41 f_{n+4}-7 f_{n+5}\right) \\
& -\frac{x_{n}}{72 h^{2}}\left(45 f_{n}-154 f_{n+1}+214 f_{n+2}-156 f_{n+3}+61 f_{n+4}-10 f_{n+5}\right)-\frac{x_{n}^{4}}{144 h^{5}}\left(f_{n}-5 f_{n+1}+10 f_{n+2}\right. \\
& \left.-10 f_{n+3}+5 f_{n+4}-f_{n+5}\right) \text {. } \\
& a_{4}=\quad \frac{5}{32 h^{2}} f_{n}-\frac{77}{144 h^{2}} f_{n+1}+\frac{107}{144 h^{2}} f_{n+2}-\frac{13}{24 h^{2}} f_{n+3}+\frac{61}{288 h^{2}} f_{n+4}-\frac{5}{144 h^{2}} f_{n+5} \\
& +\frac{x_{n}^{2}}{48 h^{4}}\left(3 f_{n}-14 f_{n+1}+26 f_{n+2}-24 f_{n+3}+11 f_{n+4}-2 f_{n+5}\right)+\frac{x_{n}}{96 h^{3}}\left(17 f_{n}-71 f_{n+1}\right. \\
& \left.+118 f_{n+2}-98 f_{n+3}+41 f_{n+4}-7 f_{n+5}\right)+\frac{x_{n}^{3}}{144 h^{5}}\left(f_{n}-5 f_{n+1}+10 f_{n+2}-10 f_{n+3}+5 f_{n+4}\right. \\
& \left.-f_{n+5}\right) \text {. } \\
& a_{5}=\quad \frac{-17}{480 h^{3}} f_{n}+\frac{71}{480 h^{3}} f_{n+1}-\frac{59}{240 h^{3}} f_{n+2}+\frac{49}{240 h^{3}} f_{n+3}-\frac{41}{480 h^{3}} f_{n+4}-\frac{7}{480 h^{3}} f_{n+5} \\
& -\frac{x_{n}}{120 h^{4}}\left(3 f_{n}-14 f_{n+1}+26 f_{n+2}-24 f_{n+3}+11 f_{n+4}-2 f_{n+5}\right)-\frac{x_{n}^{2}}{240 h^{5}}\left(f_{n}-5 f_{n+1}+\right. \\
& \left.10 f_{n+2}-10 f_{n+3}+5 f_{n+4}-f_{n+5}\right) \text {. } \\
& a_{6}=\quad \frac{1}{240 h^{4}} f_{n}-\frac{7}{360 h^{4}} f_{n+1}+\frac{13}{360 h^{4}} f_{n+2}-\frac{1}{30 h^{4}} f_{n+3}+\frac{11}{720 h^{4}} f_{n+4}-\frac{1}{360 h^{4}} f_{n+5}+ \\
& \frac{x_{n}}{720 h^{5}}\left(f_{n}-5 f_{n+1}+10 f_{n+2}-10 f_{n+3}+5 f_{n+4}-f_{n+5}\right) \text {. } \\
& a_{7}=\quad-\frac{1}{5040 h^{5}}\left(f_{n}-5 f_{n+1}+10 f_{n+2}-10 f_{n+3}+5 f_{n+4}-f_{n+5}\right) \text {. }
\end{aligned}
$$

\section{Appendix B}

$$
L=\left(\begin{array}{l}
\sum_{m=0}^{\infty} \frac{h^{m}}{m !}-2 \sum_{m=0}^{\infty} \frac{(2 h)^{m}}{m !}+\sum_{m=0}^{\infty} \frac{(3 h)^{m}}{m !}+\frac{h^{2}}{240} y_{n}^{\prime \prime}-\sum_{m=0}^{\infty} \frac{h^{m}}{(240 * m !)}\left(24(1)^{m}+194(2)^{m}+24(3)^{m}-(4)^{m}\right) \\
\sum_{m=0}^{\infty} \frac{(4 h)^{m}}{m !}+\sum_{m=0}^{\infty} \frac{(2 h)^{m}}{m !}-2 \sum_{m=0}^{\infty} \frac{(3 h)^{m}}{m !}-\sum_{m=0}^{\infty} \frac{h^{m}}{\left(240^{*} m !\right)}\left(-(1)^{m}+24(2)^{m}+194(3)^{m}+24(4)^{m}-(5)^{m}\right) \\
\sum_{m=0}^{\infty} \frac{(5 h)^{m}}{m !}+2 \sum_{m=0}^{\infty} \frac{(2 h)^{m}}{m !}-3 \sum_{m=0}^{\infty} \frac{(3 h)^{m}}{m !}-\frac{h^{2}}{240}-\sum_{m=0}^{\infty} \frac{h^{m}}{(240 * m !)}\left(-8(1)^{m}+62(2)^{m}+392(3)^{m}+257(4)^{m}+16(5)^{m}\right)
\end{array}\right)
$$

